INTERNATIONAL DESIGN CONFERENCE - DESIGN 2018

https://doi.org/10.21278/idc.2018.0190

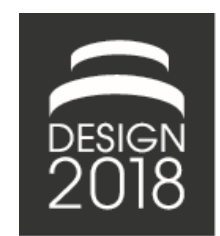

\title{
CHOOSING THE RIGHT TOOLS AND PRACTICES TO DESIGN A KNOWLEDGE MANAGEMENT SYSTEM IN A SME
}

\author{
E. Tapissier, F. Mantelet and A. Aoussat
}

\begin{abstract}
The aim of this paper is to propose a knowledge management system (KMS) design methodology for small and medium enterprises (SMEs) that address the problems encountered on the existing ones, such as the high amount of resources or the KMS sustainability. To solve these problems, we created a KMS design methodology that uses the SMEs characteristics, needs and problems to select adapted KM tools and practices and offer a tailor-made KMS. This methodology was used to design a KMS for a hightech SME and write a set of requirements to ease its implementation and ensure its sustainability.
\end{abstract}

Keywords: knowledge management, knowledge management systems, small and medium size enterprise (SME), design methodology

\section{Introduction}

Knowledge management (KM) usefulness in organizations of any size has already been demonstrated multiple times. The body of research shows that implementing KM allows companies to obtain multiple benefits such as a greater growth, better reactivity, innovation capabilities enhancements, greater efficiency in processes and procedures, and market shares gains (Nonaka and Takeuchi, 1995; Sharif et al., 2005; Lee et al., 2008; Meroño-Cerdán, 2008; Sparrow, 2011; Durst and Edvardsson, 2012; Zieba et al., 2016;). Knowledge management systems (KMS) are widespread in large companies, for which abundant literature on implementation exists (Zieba et al., 2016). However, studies suggest that Small and Medium Enterprises (SMEs) do not manage their knowledge the same way large firms do, thus making systems and concepts suitable for large companies such as knowledge engineering based practices like MOKA or CommonKADS unusable (Zieba et al., 2016). Even though knowledge is a key element for SMEs (Coyte et al., 2012; Dotsika and Patrick, 2013), literature on KM in SMEs is scarce, especially on subjects such as KMS implementation (Curran and Blackburn, 2001; Sparrow, 2011; Guthrie et al., 2012; Marzo and Scarpino, 2016; Massaro et al., 2016; Zieba et al. 2016; Cerchione and Esposito, 2017) despite the fact that SMEs make up $99.8 \%$ of the world enterprises and employ more than $67 \%$ of active workers (OECD, 2005; Airaksinen et al., 2015). In term of numbers, BenMoussa (2009) state that only 16\% of KM implementation attempts in SMEs are successful, because of the lack of suitable KMS. The problems cited are often related to too much time and resource consumption (Wong, 2005) and failure to maintain KM on the long run (BenMoussa, 2009). Since the 2000s scholars started working on KM implementation in SMEs, as well as on KM strategies, tools and practices suitable for SMEs. But as stated by Durst and Edvardsson (2012), this field of research is young and requires more attention.

Performing a literature study highlighted the fact that applying the existing large firms KM models to SMEs does not work (Wong and Aspinwall, 2004; Desouza and Awazu, 2006; Chan and Chao, 2008; BenMoussa, 2009; Supyuenyong et al., 2009; Massa and Testa, 2011; Sparrow, 2011), because SMEs 
are not scaled down large companies. Thus, using a KMS made for large firms does not mean it will perform well in a SME (Desouza and Awazu, 2006). SMEs have characteristics that make them different from large firms (Wong and Aspinwall, 2005), as well as unique regarding to each other (Curran and Blackburn, 2001). Sparrow (2011) explains that compared to large firms, SMEs have a turbulent environment, resulting in difficulties in maintaining a long-term strategy; more immediate organisationwide interaction, trust and communication; a narrower range of technical expertise; lower technological needs; and resource constraints. They also focus more on personalization, rather than codification like large firms do (Sparrow, 2011). Yet, research on this subject remains mostly theoretical, and lacks means to take the SME's need in account (Wong, 2005). There are currently only few links between literature hypotheses and KM practices in SMEs (Coyte et al., 2012; Massaro et al., 2016). It is thus necessary to take into account the usability of the KMS, as well as SMEs needs.

The purpose of this work is to implement a KMS in a high technology SME. It aims to create a tailormade KMS for a firm, and, on a wider scale, provide a methodology for any SME wishing to implement a light and easy to use KMS. The key concepts of this work are taken from multiple literature findings:

- Firms' specificities must be taken into account. This allows to make the right decisions during the design of the KMS and ensure that the resulting system is adapted to the enterprise (Wong, 2005; Massa and Testa, 2011; Sparrow, 2011).

- The KMS design complexity must be low. By guiding the user through the KMS design process, it will be easier and accessible to every SME (Wong, 2005).

- SMEs resources must be taken into account. By using only the right tools and avoiding unnecessary functionalities, the complexity and the time needed to use the KMS is reduced. By keeping the time needed to operate the KMS low, users will more likely continue to use it, thus minimizing risks of KMS desertion. The ability of the firm to use the KMS with its available resources is ensured by taking resources into consideration during the design phase (Wong, 2005; Sparrow, 2011; Durst and Edvardsson, 2012).

- The KMS must be complete in order to be functional. It is thus necessary to address the $4 \mathrm{KM}$ processes, knowledge creation, storage, transfer and use (Kanat and Atilgan, 2014; Durst and Edvardsson, 2012), during the KMS design (Wong, 2005; Hariharan, 2005; Conley and Zheng, 2009).

- The design methodology needs to answer to how to practically carry out KM in the firm. This can be done by providing guidelines and examples of KMS specifications and change management (Wong, 2005; Sparrow, 2011).

To achieve this, a methodology to design tailor-made KMS was designed: It starts with a description and a characterisation of the SME, thanks to important characteristics of SMEs found in the KM literature. This description is then used to make an assessment on the firms' readiness level and the amount of work that needs to be done to implement KM. A needs and problems analysis is then performed in the firm to understand the expected aim of the KMS, allowing the design of the specifications for a tailor-made KMS, and the choice of the right set of KM tools and practices. Change management is then performed to implement the KMS in the SME, were it will be improved through iterations to make it sustainable.

The aim of this paper is to present the KM tools and practices selection step of this KMS design methodology. This paper is organized as follows: section two focuses on a literature review focusing on the analysis of the existing KMS and KM tools, as well as SMEs KM related characteristics. Section three introduces the KMS design methodology. Section four describes how the enterprise audit is performed. Section five describes how the gathered data is used to choose need-fitting KM tools and practices for the firm. Section six describes a practical use of the methodology in a high-tech SME. Section seven concludes on this work and suggests future research directions.

\section{Literature review}

\subsection{SMEs characteristics}

To better understand how KM works in SMEs, it is necessary to understand what factors impact it in SMEs. A previous work on this subject by Tapissier (2017) identified 96 unique characteristics that were sorted in 4 categories, according to their use for KMS design. 
The "supporting" category gathers characteristics facilitating KM implementation and use in the firm. These elements, relating directly or indirectly to KM, ease the KM implementation. They might already exist in the firm before the design of the KMS. Specificities determined thanks to these characteristics makes it possible to draw a whole picture of the firm's valuable elements for KM and add the "missing" ones to the firm's processes (e.g. adding an IT communication tool in a firm that does not use one). In the end, these elements will be leveraged to enhance the adaptation and the effectiveness of the KMS, as well as making its implementation easier. A readiness assessment of the firm preparation for KM introduction and use can also be performed by using these characteristics.

The "existing" category gathers characteristics based on the firm's existing processes and KM related practices. They can be used to identify if $\mathrm{KM}$ related work is already being done, even if it is not identified as KM in the firm. Leveraging these practices in a KMS is interesting as employees won't need additional training to use them. If the SME already uses KM tools, it can be useful to study how employees perceive and use them, as it provides practical feedback. To sum up, these characteristics will facilitate the implementation of the KMS in the firm's workflow.

The "KM nature" category gathers characteristics that have a direct impact on how the KMS is built, its processes, its actors, etc. These characteristics give useful data to better define the real firms' need, allowing to create a tailor-made operating part for the KMS. It consists of SMEs' specificities that give them their uniqueness and that needs to be taken into account to create a KMS that best suits their needs. These are elements such as contextual data; operational related elements; the firm's culture; and the firm's KM needs. Studying these characteristics allows the description of the firm's specificities, which can then be used to design the right KMS strategy and select the right tools and practices.

Identifying the firm's needs makes it possible to obtain a KMS that strictly fits them, without any unnecessary elements, and takes into account each of its important specificities. Thanks to this, a smaller amount of resources is needed during the design, the implementation and the use of the KMS.

Critical Success Factors and Critical Failure Factors (CSFs and CFFs) of KM are areas that must receive attention during the KMS design, implementation and use, in order to guarantee its success (Saraph et al., 1989). They can be used to know if it is necessary to make changes in the firm and they give indications on its readiness level for KM implementation. If many CSFs/CFFs are not acceptable in the studied SME, more work will have to be done to increase the success chances of the KMS. CSF/CFFs will allow the detection of areas of the firm that need to receive attention or require action to ensure a successful KMS implementation.

These characteristics are useful to perform a precise and comprehensive description of the firm on KMimportant aspects. They can be used to provide an enterprise readiness statement (Mahmod et al., 2013); to define the changes needed to ensure a functioning KMS; to determine the KM-related needs and problems of the firm; to assist an individual from outside the company, such as a KM expert, understand its organization; and to provide decisional elements during the KM strategy design and the tools and practices selection. Studying these characteristics allows the design of the tailor-made part of the KMS.

\subsection{KM tools and practices}

A KMS is a set of practices, tools and activities allowing to achieve KM. In order to implement one in the studied firm, different existing KMS, as well as KM tools and practices were reviewed. According to Cerchione and Esposito (2017), KM practices are defined as a set of methods and techniques to support the organizational process of KM activities, and KM tools are the specific, often IT based, systems supporting KM practices. A literature study was performed to list simple and easy to use KMS, tools and practices suitable for SMEs, and then focused on more complex and integrated practices and systems, commonly used in large firms or to achieve a very specific knowledge capitalization goal. It allowed the identification of multiple KMS for SMEs, but as Wong (2005) underlines it, they come with multiple drawbacks. They provide no clear answer on how to achieve KM, assume a vast availability of resources, are very complex to use, or does not support some of the usual KM activities. It was thus necessary to find the right set of KM tools and practices usable in SMEs to design a sustainable KMS while addressing these problems. The literature study also yielded $84 \mathrm{KM}$ tools and practices (Alavi and Leidner, 2001; Fink and Ploder, 2009; Holland and Dawson, 2011; Massa and Testa, 2011; Cerchione and Esposito, 2017). KM is not always the main purpose of these tools and practices, but, as Holland 
and Dawson (2011) underline it, they can still be used to address KM problems and activities. It also allowed the identification of $21 \mathrm{KM}$ methodologies to achieve more complex activities, such as knowledge elicitation (MASK, MOKA, CommonKADS), knowledge databases creation (CommonKads, MOKA, MEREX), or case-based reasoning (CBR).

Each tool and practice of the list was studied in order to describe them and understand how to use them as well as their goals in a KM perspective. Through two brainstorming sessions, CSFs were used to exclude tools and practices that were not suitable for SMEs, such as those needing too much time, human or financial resources, as using moderate resources is a major critical success factor (Wong and Aspinwall, 2005). The current database contains 80 tools and practices relevant in SMEs. These elements are classified into 4 categories depending on what KM activities they support, which makes it possible to focus on a particular activity during the selection.

Because of the numerous existing KM tools and practices, it is hard to choose the right ones, to know if they are suitable to the needs of the firm or if it will be able to use them. This is why multiple tools and practices selection systems were created, such as AHP (Grimaldi and Rippa, 2011), the House of KM Tools Selection (HoKMTS) (Holland and Dawson, 2011), or fuzzy logic methodologies (Büyüközkan et al., 2016). They try to solve this selection problems but are complex to set up and use without preliminary training. The methodology presented in this paper offers solutions to assist the user during the KM tools and practices selection process while keeping the complexity low.

In order to produce a tailor-made KMS, as recommended in the literature (Curran and Blackburn, 2001; Wong, 2005; Wong and Aspinwall, 2005), it is necessary to choose the tools and practices and design the KM strategy according to the enterprise characteristics. The main hypothesis of this work is that, by taking into account the right characteristics and the needs of the SME, it is possible to choose the right tools and practices to guarantee a sustainable and functional KMS.

\section{Overview of the design methodology}

To obtain a suitable KMS for a firm, all the elements that might have an impact on how KM will be done must be taken into account. KM literature reveals multiple important points. By taking them into account from the beginning of the KMS design, a better suited, functional and sustainable KMS for the enterprise could be obtained. To facilitate this design phase, a 3 steps methodology was created (Figure 1).

The first step (SI) consists in a study of the SME to gather KM-relevant data usable to create a resource efficient, functioning and sustainable KMS. It is based on the literature study of KM and KMS done at the beginning of the study and makes it possible to take the findings and recommendations on KM in SMEs into account during KMS design (SI.1); to establish an enterprise description regarding to the important characteristics; to study its KM related needs and problems; and to make a readiness assessment for KM implementation (SI.2). This output is then used to establish a first set of requirements for KM (SI.3), partly specific to the firm, and to determine the required resources investments. This data will then be used during the second development step of the KMS (SII).

The second step (SII) is divided in 2 parts: The first one (SII.1) consists in practically preparing the firm for operational KM based on findings and recommendations of the literature, as well as CSF studied during the first phase. This will allow the SME to implement the necessary adaptations identified during the literature study (SI.1) and to prepare for KM use. This part is generic as the theoretical requirements for KM are the same in all SMEs. The second part (SII.2) consists in selecting the right tools and practices to implement in the firm to meet its needs and solve the problems identified during the first step (SI.2). It allows the customization of the KMS and leads to a set of requirements for the selected tools and practices. The rest of this paper will focus on this part. The second step ends with the creation of a list of functional requirements for the KMS. These specifications come from the firm preparation to meet KM needs (SII.1) as well as from the tools and practices selected (SII.2).

The third step (SIII) consists in realizing, implementing and ensuring the sustainability of the KMS. It includes employees, managers and the firm owner training to use the KMS, the implementation of the tools and practices selected during SII.2, and the promotion of their use in order to initiate a change into the enterprise organization and habits. If new KM needs appear, this step also allows iterations to add new functionalities or to remove unused features. This part will be addressed in future works. 


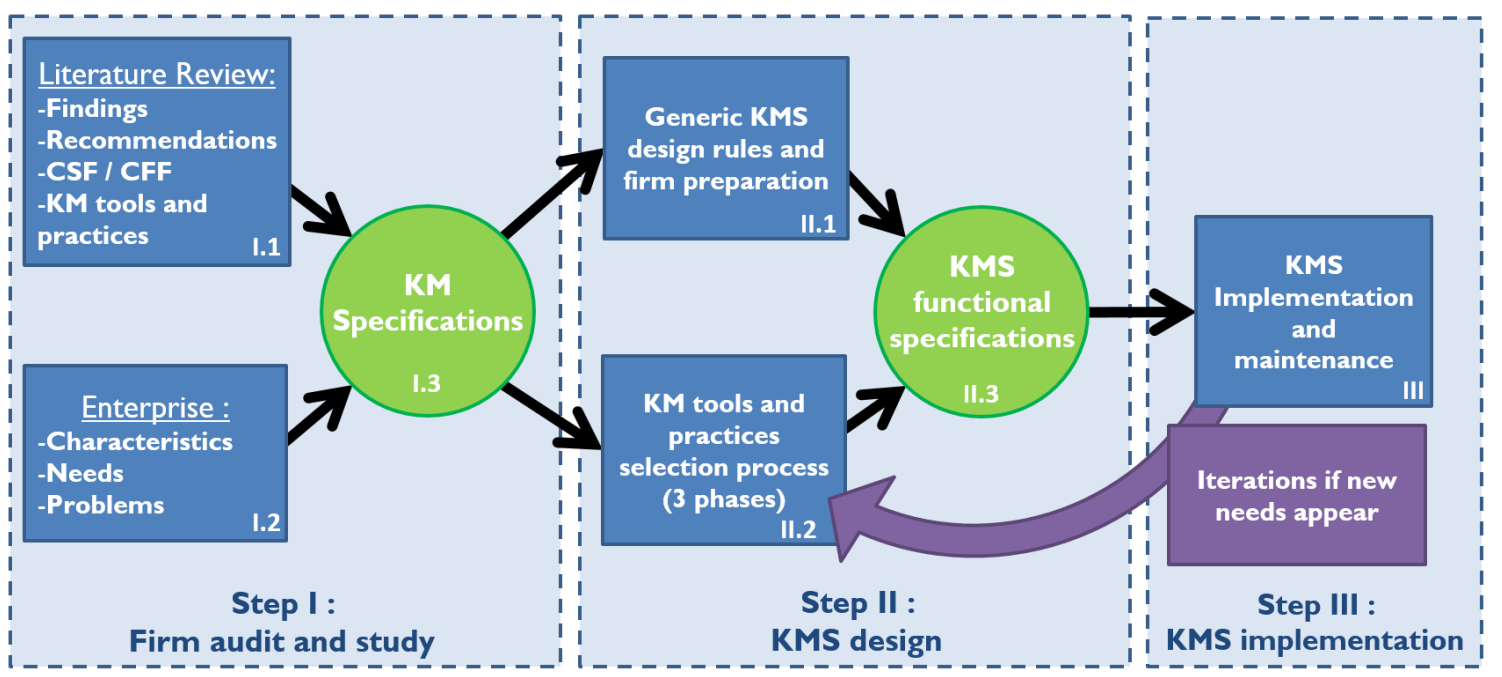

Figure 1. Proposed KMS design methodology

These three steps must be executed in the right order, as outputs are the next steps' inputs. After a short description of the first step, the paper will focus on the tools and practices selection for a SME (SII.2).

\section{First step: Enterprise audit}

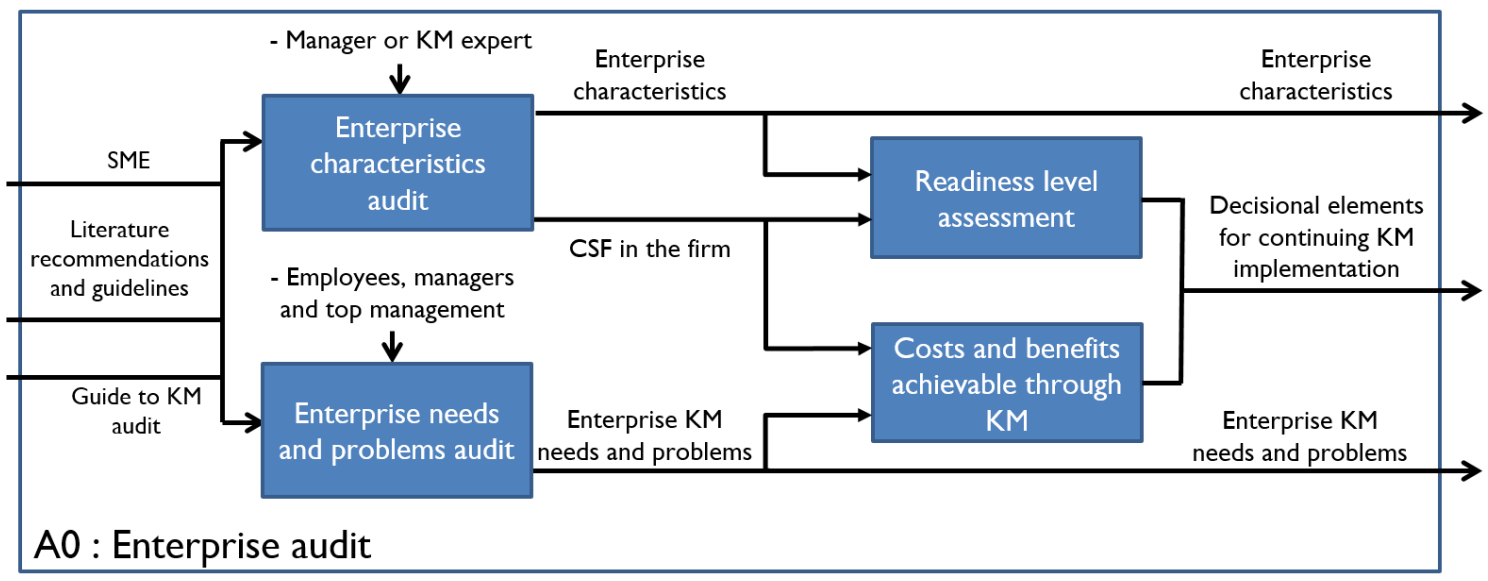

Figure 2. SADT A0 diagram of the practical part of the enterprise audit

As stated above, the first step of the methodology consists in a theoretical part and a practical part. The theoretical part is based on literature findings, recommendations and guidelines on applying KM in SMEs. These elements, deemed applicable to every SME, must be taken into account to guarantee the success of the KM strategy of the firm and leads to a first list of specifications for KM implementation. The practical part is based on the SME and its operations (Figure 2). The element studied here varies in each SME and allows the design of the "tailor-made" part of the KMS. It consists in an audit of the important characteristics described in the literature review (Section 2.2) and of the enterprise needs and problems through multiple semi-structured interviews with top management and employees of the firm. To help the user through this phase, a user guide gathering the different interesting points and questions that need to be asked in order to fully describe the firm for KM purposes is proposed. This guide takes the shape of a table gathering the different characteristics and allows the user to proceed to the SME audit without prior KM in SMEs training (Tapissier, 2017), and a list of questions to help the user find the needs and the KM related problems of the firm. The output of this part is a description of the SME regarding KM important characteristics, as well as its needs and problems. This data will allow the user to assess the readiness level of the firm for $\mathrm{KM}$ implementation and usage (and the potential 
reorganization costs), and problems that KM could solve in the SME (and the potential benefits). It is thus possible to conclude on the interest of continuing the KMS design work.

Elements from both these two parts will then be gathered together to propose a first set of KM specifications for the SME and will be reused to provide decisional elements during the selection of the KM tools and practices that will be implemented in the SME.

\section{Using the characteristics to find suitable tools and practices}

\subsection{Introduction}

Once the first step is completed, the collected data on the SME can be used to design a KM strategy. As stated above, the literature guidelines will be used to set up organizational elements to ensure the KMS success (SII.1). As this is not in the scope of this paper, it will not be presented here. The data will also be used as a support for the selection of suitable KM tools and practices part (SII.2) by providing relevant decisional insights.

The selection phase starts with the list of $80 \mathrm{KM}$ tools and practices usable in SME that was constituted according to the literature. By using it, as well as literature guidelines, findings and recommendations, the SME's problems, its needs and its description, the user can select the most suitable tools and practices for the firm. This step by step selection method answers to the fact that finding the right tools for a firm is complex because there are many existing ones and it is difficult to know them all; and that existing selection methodologies such as AHP, Fuzzy logic or HoKMTS are too complex to use for SMEs without prior training.

The selection methodology contains 3 phases, and calls upon a KM expert, a top management employee (or the owner of the SME) and employees from the domain in which KM will be used. These actors make it possible to have a comprehensive view of the SME and its organization. The SADT diagrams (Figure 3 and Figure 4) summarize the resources and the actors involved in each selection phase.

Inputs :

-KM tools and practices database

-Literature findings and guidelines

-Enterprise characteristics

-Enterprise KM needs and problems

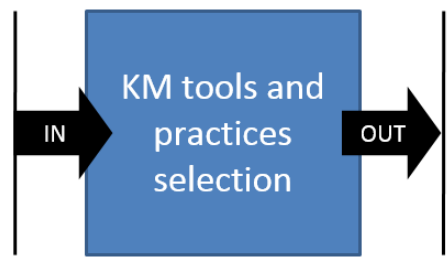

Outputs :

-Firm adapted tools and practices (list IV) chosen for implementation

-KMS specifications

Figure 3. Diagram of the KM tools and practices selection

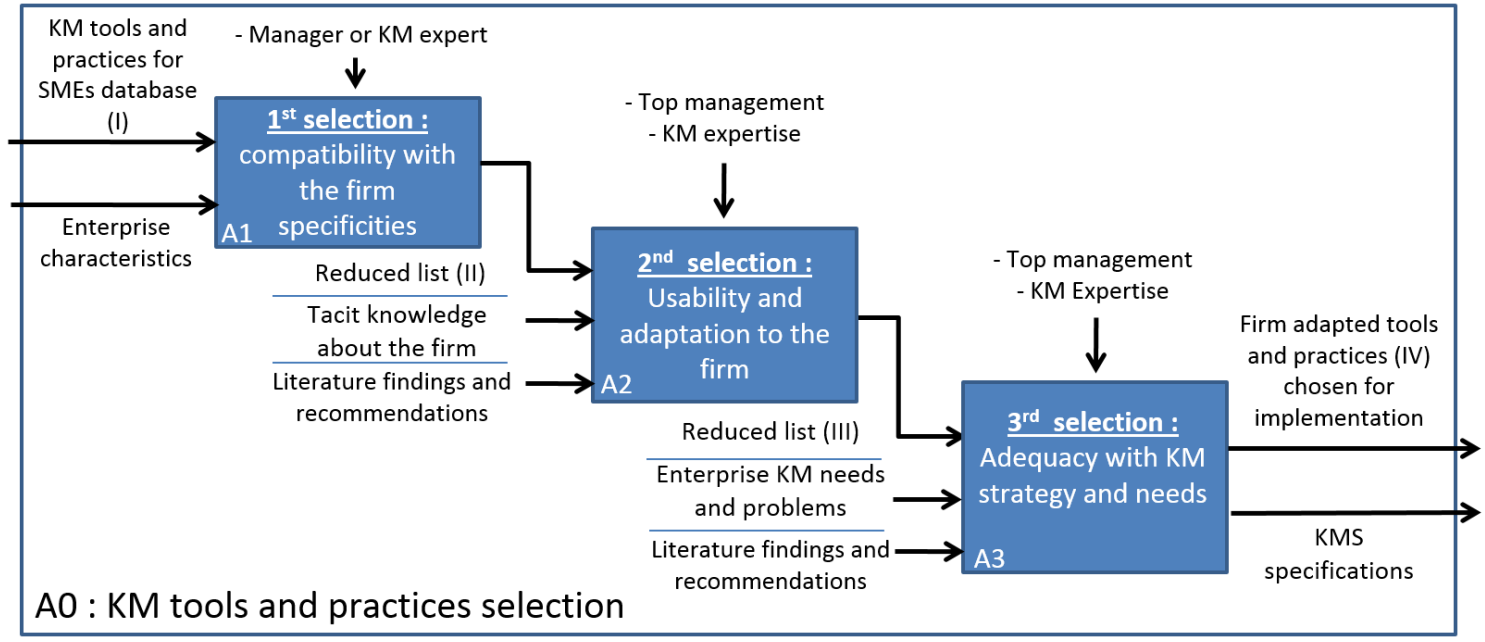

Figure 4. SADT A0 diagram of the KM tools and practices selection 


\subsection{Description of the 3 steps}

\subsubsection{Phase 1: First selection, compatibility of the tools and practices with the firm specificities}

The first phase consists in verifying if the tools and practices of the database are usable in the firm by using the result of the characteristics audit. "Nature" and "Existing" characteristics are particularly useful for this phase. Only the tools and practices that could be used in the firm are kept.

To proceed through this first review, each tool or practice is successively opposed to the different characteristics of the firm. If a tool or a practice seems to suit the firm, it is kept, and the ones that are not compatible with multiple characteristics of the firm or not likely usable in the firm are discarded. When it is unclear if an element must be removed or not, it is kept, as it will be removed during the later selection phases if it wasn't suitable. During this phase, the selection criteria are mainly formal and are based on knowledge of the enterprise and its characteristics. Its advantage lies in the fact that it can be done by anyone, as long as they have knowledge of the SME or about KM, because input elements are defined during the enterprise audit and the analysis can be done procedurally. To test this, the audit was performed by a manager of the firm and a KM expert separately, and the results were close on most characteristics. Because of the number of tools and practices, this review can be time consuming. To make it quicker, each element comes with a short description. This allows to sort the tools and practices rapidly, and to further develop the description during the second and the third selection, where less tools and practices will be studied. This selection can be done multiple times to reduce the list even further.

Selection criteria examples:

- Allowable time and financial resources: It is necessary to avoid costly and complex tools if the company can't afford them (installing an ERP in a 5-person start-up won't be sustainable in most of the cases).

- Day to day or long-term results expected from the KMS: Some of the tools can only be used to provide long term KM elements (combination of technologies within a new product) or day to day KM elements (solving problems on a production line faster). The tools and practices must be chosen accordingly.

This way, a reduced tools and practices list containing elements theoretically suitable for the firm is obtained. It will be used during the next selection phase.

\subsubsection{Phase 2: Usability and adaptation}

This selection phase consists in verifying if the tools and practices kept during phase 1 can be used in the SME. Based on tacit knowledge on the firm, such as the working habits and the organization of the firm, as well as literature recommendations, the user ponders the favourable and unfavourable elements for the use of each tools and practices in the enterprise.

This selection takes the form of a meeting with at least one person from top management (the owner, the CTO, managers) with good knowledge of the firm operations and a KM expert to assist in the understanding and the selection of the tools and concepts. It can also include other employees to offer several points of view of the firm. The criteria used are mainly informal and rely on tacit knowledge on the firm that the top management and the employees have. After presenting all tools and practices, the KM experts asks the employees to think about the potential uses of the tools in the firm by giving application examples. They are then asked to conclude if they could be interesting or not. The presentation of each tool must be short (e.g. one PowerPoint slide), to avoid giving unnecessary details and save time.

Decisional elements can be based on factors such as the supporting characteristics, the enterprise habits, its previous experience with the tools and practices, its infrastructure, its organization or the available technological means. The main goal is to keep the tools and practices that are compatible with the employees' daily activities, causing as little disruption as possible.

Selection criteria examples:

- Employees don't use computers in their activities: All the tools that need computers can be removed from the database if the equipment costs are too high for the firm or if they don't fit in the work process. 
- No experience of the employees with specific software: Software tools such as ERPs needing an extensive training could be removed from the database if the firm has no experience with them.

- Presence of emerging KM practices in the firm: practices already in use in the firm but not necessarily formalized should be kept in the database.

At the end of the meeting, a list of tools and practices suitable for the enterprise is obtained. Reducing the length of the database is important, as less tools will have to be presented in details during the last phase. This way, the time needed to select the right set of tools and practices is reduced in comparison to a selection straight from the initial database.

\subsubsection{Phase 3: Final tools and practices selection}

This last selection step consists in choosing only the tools and practices to implement in the firm to answer its KM needs. Through a meeting with top management and a KM expert, each tool kept during the second selection as well as potential use cases in the firm are presented in depth. With the advice of the KM expert, the top management can select a set of tools and practices that covers knowledge creation, storage, transfer and use, which they think is best suited to the firm.

This selection is formal as the KMS needs to be comprehensive and to answer to the firm's needs and problems. It can be validated using a conformity matrix. It is also informal as the choice remains tacit since it is based on the likability of tools and concepts to function in the firm. As the top management knows the company well, it is aware of the needs and specificities that might not have been expressed. At the end of this selection phase, a reduced list of tools and practices that must be implemented in the firm, as well as their use strategy are obtained. The goal is to keep as less elements as possible to reduce the redundancies of KM activities covered and resources needed to set up and operate the KMS.

\subsection{Tools and practices validation}

Validation criteria for the selected set of tools and practices are deeply linked to the enterprise organization, its operations and its needs and problems. Thus, it is hard to define a specific list of mandatory criteria to obtain a functioning KMS in the end. Literature still offers some guidelines and recommendations: The set of tools and practices must allow the creation of a comprehensive KMS, covering the 4 main KM activities; They must answer to all the firms KM needs and problems; The tools must be suitable to the firm specificities and likely usable in its activities; Employees having prior knowledge or training on the tools and practices is beneficial, as less training will be required; The set of tools and practices must consume as few resources as possible to avoid being deserted by employees (too much time needed to use them, high cost of implementation, etc.). This list of criteria is incomplete and could be improved. It can also be used during the third phase of the tools and practices selection to guide the choices.

If the tools and practices obtained after the second selection does not meet the requirements of the formal part of the third selection, it is possible to study again tools and practices removed during the previous steps. Selecting these tools must be done with caution. As they might be less suitable for the firm, the effort needed for their implementation could be greater and could require more employee training, or modification of the enterprise habits, technological means (e.g. IT equipment) or organization. This case was not encountered yet when using this methodology.

\subsection{Combination of tools and practices}

As suggested by Holland and Dawson (2011), tools and practices can be used in combination with each other in order to resolve the deficiencies they could present to deal with different kinds of knowledge problem. Thus, it could be interesting to figure out if such combination could be beneficial by making the tools and practices more usable and coherent, consuming fewer resources or by removing eventual unneeded functions. For example, activity redundancies between multiple tools could be removed. Following these considerations, the implementation of the selected tools and practices in the firm can start with a state of the art phase on the tools and concepts in order to set up the KMS specifications. This methodology was used in a high technology SME to select multiple tools and practices and set up the specifications for a KMS. This case study is presented in the following part. 


\section{Case study of an SME}

\subsection{Application of the methodology in a high-tech SME}

The SME in which the methodology was used designs embedded systems based on a breakthrough innovation. These products are still being improved to enhance their performance. The enterprise is very similar to a design office and only employs engineers in its technological and technical domain. In 9 years of existence, it grew to 30 employees and $4 \mathrm{~m} €$ in annual turnover. Its main KM related problems come from difficulties to find the research works carried out during the improvement of the technology. These projects are usually conducted when they could allow performance gains, but, because of the necessary implementation time and the inertia of the development of embedded systems in the company, it sometimes takes between 6 months and 2 years before they are used in the products. This results in knowledge loss in the projects, or even forgetting some of the research projects were ever done. Several problems then occur: documents are hard to find, the employees who did the studies sometimes left the firm, the studies are sometimes incomplete or not easily reusable because it relates to a technology that has evolved in the meantime. It also happened that a study on a subject was re-launched 2 years later without reusing the existing work. This results in a loss of time and resources that could be avoided.

The methodology started with an audit of the firm characteristics, as described above (Section 4), thanks to the characteristics table (SI.2). This description was done through a series of questions during a structured interview with an employee from the top management (for about 2 hours). The needs and problems analysis was done through 6 semi-structured interviews with employees from top management, engineers and project managers in charge of the different enterprise domains (about 45 minutes per interview). For more information on this audit, please refer to Tapissier (2017). This audit provided a description of the company regarding to the important characteristics for KM previously defined, as well as a list of problems, needs and objectives to address through KM implementation.

Based on this description of the firm (SI.2) and literature findings, recommendations and guidelines (SI.1), specifications to achieve KM in the SME were proposed (SI.3). They include 58 requirements relating to CSF, recommendations and findings, and 15 requirements relating to the company's needs and problems. For example, problems linked to loss of knowledge objects in the firm, such as the research projects described above, were translated to the following requirement: "The KMS must allow to find and access the work already done that could be reused on other projects". These requirements were then used during the tools and practices selection phase to allow a better understanding of the firm's needs. This case study continued with the tools and practices selection (SII.2).

To proceed with the first phase of the tools and practices selection, a researcher first re-read the data from the firm audit to remember the specificities identified. The researcher then used the description of each element to determine if the elements from the tools and practices database (list I, initially containing 80 tools and practices) were compatible with the enterprise and likely usable within it. By going through the list 3 times and removing the tools and practices deemed inadequate, 18 tools (list II) that could ensure a functioning KM in this SME were kept.

For the second phase, the researcher worked with a project manager who has been in the firm since its creation. During a meeting, each of the 18 tools from list II were presented using one PowerPoint slide containing information such as knowledge activities supported, principles, potential usage and examples. The project manager suggested the exclusion of 10 tools and practices because they required too much resources or employee training, or were inadequate to the firm's needs. The selection yielded 8 reasonably usable tools for the SME (list III): Process mapping, knowledge elicitation, lessons learned, after action reviews, best practices, wikis, knowledge mapping and knowledge modelling.

For the third phase, the researcher, the same project manager and the CTO of the company worked together. After presenting the 8 tools and practices with more details and examples, a combination of wiki, knowledge mapping and knowledge modelling was selected, as it answered to all the firm's needs. These 3 tools and practices are currently being implemented in the firm.

\subsection{Selection outcome}

This selection is satisfactory as the chosen tools and practices validate several validation criteria defined above (Section 5.3). They allow a comprehensive KMS as the $4 \mathrm{KM}$ activities are covered, the tools are 
reasonably usable in the firm, where everyone uses a computer; some employees have prior knowledge of wikis as they already set up one; and it cost little resources as they don't need licenses to operate. Moreover, taking the firm's specificities into account allowed to answer to all of the firm's KM problems. Thus, taking into account the characteristics of the company in order to design a tailor-made KMS makes it possible to choose the right tools and practices for the firm while only going through simple and procedural phases.

The 3 selected tools and practices can be combined in a single tool in order to remove the redundancies they contain, as well as useless functions regarding to the KM strategy of the firm. This tailor-made tool will use the minimalist structure of the wiki and will use knowledge mapping and modelling to identify important knowledge. The objective is to allow users to find relevant knowledge quickly, as well as related knowledge that could be useful in addition to what they are looking for.

The wiki will be used to support information and knowledge objects, its minimal and flexible structure allowing it to store mostly every content. Knowledge mapping and modelling of the firm projects will bring out the organizational and classification structure for knowledge objects and allow to know what knowledge needs to be capitalized in articles. These first wiki articles will then be used as templates for the users as well as test means for the classification structure and the others wiki functionalities. Guidelines on how to use the wiki will then be designed and used to train the users.

Knowledge mapping will bring out the structure of the wiki and allow the identification of crucial knowledge of the firm. It will provide information on what needs to be capitalized in the wiki, as it is important for the firm, and what knowledge is not very useful and does not need to be documented, as it won't be reused by other employees. Knowledge modelling will bring out the structure of the wiki, and create connections between different projects, making it easier to find knowledge reusable from one project to another, as well as similar knowledge and concepts present in both projects.

Following the selection and the combination phase, a list of requirements for the KMS specifications was produced. These 55 requirements are related to tools and practices and ensure that the KMS will have the right tools, will be adapted to the firm and that the time required for its use will be optimized. They complete the 48 others, related to the generic KMS design rules and the firm preparation to KM implementation from the step II.1. These specifications can then be used to implement the tailor-made KMS in the firm.

\section{Conclusion}

\subsection{Limitations}

Although the first trial has yielded positive results, this tools and practices selection methodology has so far only been tested in a single SME. It will therefore be interesting to apply it in others SMEs similar to the audited company and will allow to conclude if it is functional. It also needs to be applied in SMEs with different core activities to verify its universality. Moreover, the KM tools and practices database could be improved by adding potentially interesting tools and methods, or parts of more complex tools that could be used as is, from different domains such as knowledge engineering (KE) or intellectual capital (IC). Performance of the KMS has not been measured yet, as the KMS is still being implemented. It will be done through the use of indicators, allowing the description of the enterprise before and after the implementation of the KMS.

\subsection{Conclusion and direction for future research}

By taking into account the initial KM need and the characteristics of a SME, the presented methodology allows the design of a KMS meeting the needs of the firm, both in terms of KM and adaptation, while taking resources into account. It helps the user to describe the firm, to take literature recommendations in account, and to use these elements to build a need-fitting KMS, in order to guarantee its success. The main interest of this work lies in the fact that it brings $\mathrm{KM}$ theory into practice, which is actually lacking in the literature, particularly on SMEs. By using a set of characteristics and important elements for KM in SMEs, the specificities of the SME can be included in the KMS design.

During its first use, the audit step proved to be efficient, as the firm description was deemed representative of the firm by the firm owner and provided the required information on the firm during 
the tools and concepts selection. The tools and practices selection is facilitated by the preliminary definition of the important specificities of the firm as well as the use of the tools and practices database, containing references to find more information on each tool quickly and easily. The selection process seemed more accessible than the solutions proposed in the literature to the managers of the firm. This addresses the design complexity of KMS raised by some authors. In the end, the global methodology assists the user from the expression of the KM need to the specifications and implementation of the KMS. It makes sure that the designed KMS is comprehensive and that it answers how to adopt KM in the firm. The requirements designed using the generic KMS design rules and the firm preparation are used to guide the implementation and guarantee that the KMS will be sustainable. The requirements from the KM tools and practices ensure that the enterprise needs and problems are addressed.

The development and the implementation of the KMS for the studied SME are currently in progress. Results will then be published along with the complete design methodology. Once the KMS is implemented in the SME, this work will continue through iterations on its contents to determine what adaptations are necessary and if they could have been planned during the design phase. The complete methodology will then be evaluated in another SME, similar to the one presented in this paper.

\section{References}

Airaksinen, A., Luomaranta, H., Alajääskö, P. and Roodhuijzen, A., (2015), Statistics on small and medium-sized enterprise 2015, Eurostat.

Alavi, M. and Leidner, D.E. (2001), "Review: Knowledge Management and Knowledge Management Systems: Conceptual Foundations and Research Issues", MIS Quarterly, Vol. 25 No. 107, pp. 107-136. https://doi.org/10.2307/3250961

BenMoussa, C. (2009), "Impediments to Knowledge Management", 2009 International Conference on Information Management and Engineering, IEEE, pp. 322-329. https://doi.org/10.1109/ICIME.2009.146

Büyüközkan, G., Parlak, I.B. and Tolga, A.C. (2016), "Evaluation of Knowledge Management Tools by Using an Interval Type-2 Fuzzy TOPSIS Method", International Journal of Computational Intelligence Systems, Vol. 9 No. 5, pp. 812-826. https://doi.org/10.1080/18756891.2016.1237182

Cerchione, R. and Esposito, E. (2017), "Using knowledge management systems: A taxonomy of SME strategies", International Journal of Information Management, Vol. 37 No. 1B, pp. 1551-1562. https://doi.org/10.1016/j.ijinfomgt.2016.10.007

Chan, I. and Chao, C.-K. (2008), "Knowledge management in small and medium-sized enterprises", Communications of the ACM, Vol. 51 No. 4, pp. 83-88. https://doi.org/10.1145/1330311.1330328

Conley, C.A. and Zheng, W. (2009), "Factors Critical to Knowledge Management Success", Advances in Developing Human Resources, Vol. 11 No. 3, pp. 334-348. https://doi.org/10.1177/1523422309338159

Coyte, R., Ricceri, F. and Guthrie, J. (2012), "The management of knowledge resources in SMEs: an Australian case study", Journal of Knowledge Management, Vol. 16 No. 5, pp. $789-807$. https://doi.org/10.1108/13673271211262817

Curran, J. and Blackburn, R.A. (2001), Researching the small enterprise, SAGE, London. https://doi.org/10.4135/9781849209847

Desouza, K.C. and Awazu, Y. (2006), "Knowledge management at SMEs: five peculiarities", Journal of Knowledge Management, Vol. 10 No. 1, pp. 32-43. https://doi.org/10.1108/13673270610650085

Dotsika, F. and Patrick, K. (2013), "Collaborative KM for SMEs: a framework evaluation study”, Information Technology and People, Vol. 26 No. 4, pp. 368-382. https://doi.org/10.1108/ITP-11-2012-0142

Durst, S. and Edvardsson, I.R., (2012), "Knowledge management in SMEs: a literature review", Journal of Knowledge Management, Vol. 16 No. 6, pp. 879-903. https://doi.org/10.1108/13673271211276173

Fink, K. and Ploder, C. (2009), “Knowledge Management Toolkit for SMEs”, International Journal of Knowledge Management, Vol. 5 No. 1, pp. 46-60. https://doi.org/10.4018/jkm.2009010104

Grimaldi, M. and Rippa, P. (2011), "An AHP-based framework for selecting knowledge management tools to sustain innovation process", Knowledge and Process Management, Vol. 18 No. 1, pp. 45-55. https://doi.org/10.1002/kpm.365

Guthrie, J., Ricceri, F. and Dumay, J. (2012), "Reflections and projections: A decade of Intellectual Capital Accounting Research", The British Accounting Review, Vol. 44 No. 2, pp. 68-82. https://doi.org/10.1016/j.bar.2012.03.004

Hariharan, A. (2005), “Critical success factors for knowledge management”, Knowledge Management Review, Vol. 8 No. 2, pp. 16-19.

Holland, S. and Dawson, R. (2011), "Classification and selection of tools for quality knowledge management", Software Quality Journal, Vol. 19 No. 2, pp. 393-409. https://doi.org/10.1007/s11219-010-9121-8 
Kanat, S. and Atilgan, T. (2014), "Effects of knowledge management on supply chain management in the clothing sector: Turkish case", Fibres and Textiles in Eastern Europe, Vol. 1 No. 103, pp. 9-13.

Lee, C.L., Ho, C.T. and Chiu, Y.L. (2008), "The impact of knowledge management enablers on non-financial performance in small and medium enterprises", International Journal of Technology Management, Vol. 43 No. 1-3, pp. 266-283. https://doi.org/10.1504/IJTM.2008.019419

Mahmod, R., Rosnan, H. and Hazman-Fitri, M.H. (2013), "Knowledge management and innovation readiness among SMEs in Malaysia", Business Engineering and Industrial Applications Colloquium (BEIAC), 2013, IEEE, pp. 698-702. https://doi.org/10.1109/BEIAC.2013.6560221

Marzo, G. and Scarpino, E. (2016), "Exploring intellectual capital management in SMEs: an in-depth Italian case study", Journal of Intellectual Capital, Vol. 17 No. 1, pp. 27-51. https://doi.org/10.1108/JIC-09-2015-0075

Massa, S. and Testa, S. (2011), "Knowledge domain and innovation behaviour: A framework to conceptualize KMSs in small and medium enterprises", VINE, Vol. 41 No. 4, pp. 483-504. https://doi.org/10.1108/03055721111188557

Massaro, M., Handley, K., Bagnoli, C. and Dumay, J. (2016), "Knowledge management in small and medium enterprises: a structured literature review”, Journal of Knowledge Management, Vol. 20 No. 2, pp. $258-291$. https://doi.org/10.1108/JKM-08-2015-0320

Meroño-Cerdán, A.L. (2008), "Groupware uses and influence on performance in SMEs", Journal of Computer Information Systems, Vol. 48 No. 4, pp. 87-96.

Nonaka, I. and Takeuchi, H. (1995), The knowledge creating company: how Japanese companies create the dynamics of innovation, Oxford University Press, New York, NY.

OECD (2005), SME and Entrepreneurship Outlook, OECD Publishing.

Saraph, J.V., Benson, P.G. and Schroeder, R.G. (1989), "An Instrument for Measuring the Critical Factors of Quality Management”, Decision Sciences, Vol. 20 No. 4, pp. 810-829. https://doi.org/10.1111/j.15405915.1989.tb01421.x

Sharif, M.N.A., Zakaria, N.H., Ching, L.S. and Fung, L.S. (2005), "Facilitating Knowledge Sharing Through Lessons Learned System”, Journal of Knowledge Management Practice, Vol. 12, pp. 45-57.

Sparrow, J. (2011), "Knowledge Management in Small and Medium Sized Enterprises", In: Schwartz, D. and Te'eni, D. (Eds.), Encyclopedia of Knowledge Management, 2nd ed., IGI Global, pp. 671-682. https://doi.org/10.4018/978-1-59904-931-1.ch065

Supyuenyong, V., Islam, N. and Kulkarni, U. (2009), "Influence of SME characteristics on knowledge management processes: The case study of enterprise resource planning service providers", Journal of Enterprise Information Management, Vol. $22 \quad$ No. $1-2, \quad$ pp. $63-80$. https://doi.org/10.1108/17410390910922831

Tapissier, E., Mantelet, F. and Aoussat, A. (2017), "Proposal of an adaptable, need-fitting Knowledge Management System design tool for SMEs", AIP Priméca conference, La Plagne.

Wong, K.Y. (2005), "A critical review of knowledge management frameworks", International Journal of Information Technology and Management, Vol. 4 No. 3, https://doi.org/10.1504/IJITM.2005.006785

Wong, K.Y. and Aspinwall, E. (2004), "Characterizing knowledge management in the small business environment", Journal of Knowledge Management, Vol. 8, pp. 44-61. https://doi.org/10.1108/13673270410541033

Wong, K.Y. and Aspinwall, E. (2005), "An empirical study of the important factors for knowledge-management adoption in the SME sector", Journal of Knowledge Management, Vol. 9 No. 3, pp. 64-82. https://doi.org/10.1108/13673270510602773

Zieba, M., Bolisani, E. and Scarso, E. (2016), "Emergent approach to knowledge management by small companies: multiple case-study research", Journal of Knowledge Management, Vol. 20 No. 2, pp. $292-307$. https://doi.org/10.1108/JKM-07-2015-0271

Edouard Tapissier, PhD Student

Arts et Métiers ParisTech - ENSAM, LCPI

95 rue d'albufera, 27200 Vernon, France

Email: edouard.tapissier@ensam.eu 